\title{
Análise de Agrupamento para Implementação da Meta-Análise em Estimativas de Herdabilidade para Características de Crescimento em Bovinos de Corte ${ }^{1}$
}

\author{
Juliana Di Giorgio Giannotti², Irineu Umberto Packer ${ }^{3}$, Maria Eugênia Zerlotti Mercadante ${ }^{4}$, \\ César Gonçalves de Lima ${ }^{5}$
}

\begin{abstract}
RESUMO - Estimativas de herdabilidade de características de crescimento são informações muito utilizadas em programas de melhoramento genético de bovinos de corte. Neste trabalho, foram compiladas 869 estimativas de herdabilidade, provenientes de 186 artigos publicados, das características peso ao nascimento, peso à desmama, peso aos 365 dias e peso aos 550 dias, de bovinos de corte de origem indiana. As estimativas foram divididas em grupos, em cada uma das quatro características, utilizando-se o método de agrupamento de Ward, e combinadas, dentro de cada grupo, por intermédio da meta-análise. Observou-se, para todas as características, que os grupos compostos por animais da raça Nelore presentes, em sua maioria, no Brasil, apresentaram maiores estimativas combinadas de herdabilidade que os demais grupos. Foram utilizados dois métodos, o da máxima verossimilhança restrita e o proposto por DerSimonian e Laird, para estimar a variância entre os estudos, tendo o primeiro apresentado valores superiores.
\end{abstract}

Palavras-chave: análise multivariada, estimativas combinadas, modelo de efeito aleatório, parâmetros genéticos

\section{Cluster Analysis for Meta-Analysis Implementation for Heritability of Estimates Growth Traits in Beef Cattle}

\begin{abstract}
Heritability estimates of growth traits are essential informations in animal breeding programs. In this paper, 869 heritability estimates of birth weight, weaning weight, weight at 365 days old and weight at 550 days old, of 186 reports were compiled. The estimates were divided in groups using the Ward method of cluster analysis, and pooled by meta-analysis. It was observed, for all traits, that groups from Nelore breed, majority in Brasil, showed greater pooled heritability estimates than the other groups. The methods, restricted maximum likelihood and DerSimonian and Laird, were used to estimate the variance between studies, where the first method showed higher variances.
\end{abstract}

Key Words: genetic parameters, multivariate analysis, pooled estimates, random effects models

\section{Introdução}

A herdabilidade de uma característica é a proporção da variação fenotípica resultante da variância genética aditiva existente na população. É um parâmetro essencial em programas de melhoramento genético, pois indica quanto das diferenças existentes no desempenho para uma característica são determinadas por fatores genéticos ou ambientais, tendo assim papel fundamental na predição dos valores genéticos dos animais (Bourdon, 2000). Os valores estimados dos parâmetros genéticos são característicos da amostra utilizada e podem mudar no tempo em razão da seleção e de decisões de manejo. Desse modo, as estimativas de herdabilidade $\left(\hat{h}^{2}\right)$ podem ser afetadas tanto por fatores como raça do animal e país de origem, tipo de manejo alimentar e sexo como pelo método de estimação, que é independente da população (Koots et al., 1994). Há, atualmente, grande número de trabalhos publicados contendo para diferentes características observadas nos animais, graças à importância desse parâmetro e sua ampla utilização em programas de melhoramento animal. Especificamente em melhoramento de bovinos de corte, características relativas ao peso do animal são bastante utilizadas, em virtude da facilidade de obtenção, da média herdabilidade e à relação, quase sempre alta, com o peso ao abate.

Muitas vezes, as $\hat{h}^{2}$ para determinada característica obtidas de um grupo de animais podem não representar bem o valor verdadeiro da herdabilidade, em virtude, principalmente, do tamanho reduzido do

\footnotetext{
${ }^{1}$ Parte da tese de doutorado da primeira autora. Pesquisa financiada pelo CNPq.

${ }^{2}$ Pesquisadora Científica do Instituto de Economia Agrícola - IEA, São Paulo, SP. E.mail: juliana@iea.sp.gov.br

3 Orientador e Professor do Dep. Zootecnia da ESALQ/USP, Piracicaba/SP

${ }^{4}$ Pesquisadora do Instituto de Zootecnia - EEZ Sertãozinho, Sertãozinho/SP

5 Professor do Dep. de Ciências Básicas da FZEA/USP, Pirassununga/SP.
} 
banco de dados. Portanto, combinar de trabalhos publicados, obtendo valores médios, pode ser recomendável (Koots et al., 1994).

A obtenção de estimativas combinadas por meio da meta-análise, definida por Glass (1976) como procedimento estatístico para obtenção de uma medida comum entre várias pesquisas distintas mas relacionadas, pode ser uma opção mais segura do que simplesmente obter uma média de resultados publicados. Este fato se deve aos propósitos atribuídos à meta-análise: aumento do tamanho amostral; generalizações das conclusões para um conjunto de estudos; teste da variabilidade entre os estudos; realização análise de subgrupo (Fagard et al., 1996).

A natureza diversa entre os estudos é um aspecto fundamental na meta-análise, pois quanto mais homogêneos forem os resultados a serem combinados, mais confiável será a estimativa combinada. Na implementação da meta-análise, testes de homogeneidade são formulados e, com base nos seus resultados, opta-se por modelar a variância entre os estudos, quando há ausência de homogeneidade, ou por combiná-los sem considerar a variância entre eles (Wang \& Bushman, 1999). Os métodos utilizados para estimar a variância entre os estudos são o da máxima verossimilhança restrita $(M V R)$, o proposto por DerSimonian \& Laird $(D L)$ e o Bayesiano (Normand, 1999). Algumas discussões quanto ao método empregado para estimar a variância entre os estudos podem ser encontradas em artigos como os de DerSimonian \& Laird (1986), Normand (1999) e Brockwell \& Gordon (2001).

A técnica multivariada de análise de agrupamento é uma maneira de se obter grupos homogêneos, por um esquema que possibilite reunir os dados em questão em um determinado número de grupos, de modo que exista grande homogeneidade dentro de cada grupo e heterogeneidade entre eles (Jonhson \& Wichern, 1998).

Tendo como conjunto de dados as estimativas publicadas de herdabilidade das características peso ao nascimento $(\mathrm{PN})$, peso à desmama $(\mathrm{PD})$, peso aos 365 dias (P365) e peso aos 550 (P550) de bovinos de corte de origem indiana, objetivou-se, com este trabalho, realizar a meta-análise das $\hat{h}^{2}$ para essas características, fazendo-se previamente uma análise de agrupamento para cada uma das quatro características, considerando-se como variáveis os fatores raça, país, método de estimação, número de animais e o próprio valor da estimativa de herdabilidade.

\section{Material e Métodos}

Os dados utilizados neste trabalho referem-se a $869 \hat{h}^{2}$ das características de crescimento PN, PD, P365 e P550, obtidas em populações de bovinos de corte de origem indiana (182 de PN, 331 de PD, 153 de P365 e 203 de P550), procedentes de 186 artigos publicados, dos quais 102 constam no trabalho de revisão realizado por Mercadante et al. (1995) e 84 são provenientes de pesquisa bibliográfica realizada de 1995 a 2003 para a atualização destes dados. A pesquisa supracitada englobou anais de congressos, teses, dissertações e base de dados como CAB e AGRIS, utilizando-se as palavras-chave "nelore”; "nellore”; "zebu”; "bos indicus”; e "heritabili*”.

Sempre que a variância $\left(s_{i}^{2}\right)$ de uma $\hat{h}^{2}$ não estava presente no trabalho, foi estimada por meio da metodologia descrita por Koots et al. (1994) e Falconer \& Mackay (1996).

As $\hat{h}^{2}$ foram divididas em grupos, dentro de cada uma das quatro características, adotando-se a análise de agrupamento. Procurou-se reunir dentro de cada grupo as $\hat{h}^{2}$ mais similares a partir das informações do método empregado para sua estimação, do seu tamanho amostral, da raça, do país de origem dos dados e do próprio valor de cada . Criaram-se variáveis fictícias (“dummy”) para as variáveis categorizadas (método de estimação, país e raça) e fez-se uma padronização das variáveis quantitativas (tamanho amostral e $\hat{h}^{2}$ ), resultando nas variáveis: $x_{1}$, cujos valores foram 1 , se raça Nelore, e 0 , se raça não Nelore; $x_{2}$, cujos valores foram 1 , se país Brasil, e 0 , se país não-Brasil; $x_{3}$, cujos valores foram 1 , se modelo animal, e 0 , se não modelo animal; $x_{4}$, cujos valores foram 1 , se modelo touro (famílias de meios-irmãos), e 0 , se não modelo touro; $x_{5}$ e $x_{6}$, cujos valores tanto do i-ésimo tamanho amostral quanto da $i$-ésima foram padronizados utilizando-se a expressão $z=X_{i}$-mín(x)/(máx(x)-mín(x)), em que $X_{i}$ é o i-ésimo tamanho amostral ou a i-ésima $\hat{h}^{2}, \operatorname{mí}(x)$ o menor tamanho amostral ou a menor $\hat{h}^{2}$ e máx (x), o maior tamanho amostral ou a maior . Para formar os grupos, utilizou-se o método hierárquico de Ward, cuja principal característica é compor grupos mais homogêneos (Sharma, 1996; Jonhson \& Wichern, 1998). O critério do pseudo F, em que um valor elevado desta estatística, quando comparado ao precedente, indica o ponto de parada, foi empregado para obter o número de grupos. Esta análise foi feita utilizando os 
PROC CLUSTER e TREE do SAS (SAS, 1999a; SAS, 1999b; SAS, 1999c).

De posse das $\hat{h}^{2}$ divididas em grupos, a metaanálise envolveu a análise exploratória dos dados, o teste de homogeneidade tanto dentro quanto entre os grupos, a modelagem da variação e a obtenção da estimativa combinada para os diferentes grupos (Hedges \& Olkin, 1985).

Para a análise exploratória, construíram-se gráficos de "box-plot" para os diferentes grupos nas quatro características. Nesse dispositivo, observouse a distribuição e o resumo das principais estatísticas do conjunto de dados (mediana, quartis e prováveis dados discrepantes), visando, principalmente, verificar se os conjuntos de dados são comparáveis entre si (no caso, os diferentes grupos dentro de cada característica) (Bussab \& Morettin, 2003). O teste de Shapiro-Wilk do SAS (Shapiro \& Wilk, 1965) foi utilizado para verificar se os dados seguem distribuição normal. Na ausência de normalidade, os dados foram transformados, por intemédio do algoritmo de Box e Cox, pela macro boxglm do SAS (SAS, 2002).

O teste de homogeneidade, utilizando-se as $\hat{h}^{2}$ transformadas em todos os níveis entre grupos, foi realizado formulando-se a hipótese de nulidade $H_{0}: h_{1+}^{2}=h_{2+}^{2}=\ldots=h_{g+}^{2}$, sendo $g$ o número de grupos e $\hat{h}_{i+}^{2}$, a estimativa combinada das herdabilidades do $i$ ésimo grupo versus a hipótese alternativa $H_{A}$ : ao menos uma $\hat{h}_{i+}^{2}$ difere das demais, por intermédio da estatística $Q_{\text {entre }}=\sum_{i=1}^{g} w_{i+}\left(\hat{h}_{i+}^{2}-\hat{h}_{++}^{2}\right)^{2}$, em que: $w_{i+}=\sum_{j=1}^{m_{i}} w_{i j}$, sendo $j$ o número de $\hat{h}^{2}$ de cada grupo e $w_{i j}=1 / s_{i j}^{2}$, a ponderação; $\hat{h}_{i+}^{2}=\sum_{j=1}^{m_{i}} w_{i j} \hat{h}_{i j}^{2} / \sum_{j=1}^{m_{i}} w_{i j}$; e $\hat{h}_{++}^{2}=\sum_{i=1}^{g} w_{i+} \hat{h}_{i+}^{2} / \sum_{i=1}^{g} w_{i+}$. Rejeitou-se a hipótese de nulidade, no nível de significância $p$, se $Q_{\text {entre }}$ excedeu o valor crítico de $100(1-p) \%$ da distribuição de qui-quadrado com $g$-1 graus de liberdade. Para o teste de homogeneidade dentro de grupos a hipótese de nulidade foi $H_{0}: h_{i 1}^{2}=h_{i 2}^{2}=\ldots=h_{i m_{i}}^{2}=h_{i+}^{2}$, em que $i=1,2, \ldots, g$. Utilizou-se a estatística, $Q_{\text {dentro }}=\sum_{i=1}^{k} \sum_{j=1}^{m_{i}} w_{i j}\left(\hat{h}_{i j}^{2}-\hat{h}_{i+}^{2}\right)$, em que $k=m_{1}+m_{2}+\ldots$ $+m_{g}$, com distribuição de qui-quadrado com k-g graus de liberdade (Wang \& Bushman, 1999). Estes testes foram realizados a partir das macros Within \& Wavgz (SAS, 1997), e do procedimento FORMAT (SAS, 1990). A Tabela 1 resume as várias causas de heterogeneidade numa meta-análise com $g$ grupos, assim como o teste estatístico e os correspondentes graus de liberdade.

$\mathrm{Na}$ ausência de homogeneidade entre os estudos, o modelo de efeito aleatório, adotado para a realização da meta-análise e posterior obtenção da estimativa combinada para as estimativas de herdabilidade teve como estrutura $\hat{h}_{i}^{2}=h_{+}^{2}+\varepsilon_{i}+e_{i}$, em que: $\hat{h}_{i}^{2}$ representa o valor da $i$-ésima estimativa de herdabilidade de determinada característica $\hat{h}_{i}^{2} \stackrel{\text { indep. }}{\sim} \operatorname{Normal}\left(h_{+}^{2}, \sigma_{\mathrm{i}}^{2}+\tau^{2}\right)$; $\hat{h}_{i+}^{2}$ representa o valor da estimativa combinada das herdabilidades; $e_{i}$ é o erro aleatório $\left(e_{i} \stackrel{\text { indep. }}{\sim} \operatorname{Normal}\left(0, \sigma_{i}^{2}\right)\right) ; \varepsilon_{i}$ é o erro aleatório $\left.\varepsilon_{i} \stackrel{\text { indep. }}{\sim} \operatorname{Normal}\left(0, \tau^{2}\right)\right)$. A variância entre estudos é representada por $\tau^{2}$ e foi estimada por dois métodos distintos: o $M V R\left(\hat{\tau}_{M V R}^{2}\right)$, pelo procedimento MIXED (SAS, 1997); e o $D L$, que é um método não-iterativo, tendo como estimador $\hat{\tau}_{D L}^{2}=\operatorname{máx}\left\{0,\left(Q_{\text {total }}-(k-1)\right) /\left(\sum_{i=1}^{k} w_{i}-\left(\sum_{i=1}^{k} w_{i}^{2} / \sum_{i=1}^{k} w_{i}\right)\right)\right\}$

(DerSimonian \& Laird, 1986; Normand, 1999).

Para obtenção das estimativas combinadas das $\hat{h}^{2}\left(\hat{h}_{+}^{2 *}\right)$ dentro de cada grupo, para cada uma das

Tabela 1 - Testes de homogeneidade para um modelo de meta-análise com g grupos

Table 1 - Homogeneity tests for a meta-analysis model with $g$ groups

\begin{tabular}{|c|c|c|}
\hline $\begin{array}{l}\text { Causas de } \\
\text { heterogeneidade } \\
\text { Source of } \\
\text { heterogeneity }\end{array}$ & $\begin{array}{c}\text { Estatística } Q \\
Q \text { statistic }\end{array}$ & $\begin{array}{l}\text { Graus de liberdade } \\
\text { Degrees of freedom }\end{array}$ \\
\hline $\begin{array}{l}\text { Entre grupos } \\
\text { Between groups }\end{array}$ & $Q_{\text {entre }} Q_{\text {between }}$ & $g-1$ \\
\hline $\begin{array}{l}\text { Dentro de grupos } \\
\text { Within groups }\end{array}$ & $Q_{\text {dentro }} Q_{\text {within }}$ & $k-g$ \\
\hline $\begin{array}{l}\text { Dentro do grupo } 1 \\
\text { Within group } 1\end{array}$ & $Q_{\text {dentro } 1} Q_{\text {within } 1}$ & $m_{1}-1$ \\
\hline $\begin{array}{l}\text { Dentro do grupo } g \\
\text { Within group } g\end{array}$ & $Q_{\text {dentro }}{ }_{g} Q_{\text {within } g}$ & $m_{g}-1$ \\
\hline $\begin{array}{l}\text { Total } \\
\text { Total }\end{array}$ & $Q_{\text {total }} Q_{\text {total }}$ & k-1 \\
\hline
\end{tabular}


quatro características de crescimento, utilizou-se a seguinte equação: $\hat{h}_{+}^{2^{*}}=\sum_{i=1}^{k} \sum_{j=1}^{m_{i}} w_{i j}^{*} \hat{h}_{i j}^{2} / \sum_{i=1}^{k} \sum_{j=1}^{m_{i}} w_{i j}^{*}$, em que a ponderação foi $w_{i j}^{*}=1 /\left(s_{i j}^{2}+\hat{\tau}^{2}\right)$ (Wang \& Bushman, 1999) e o desvio-padrão combinado, associado a cada uma das $\hat{h}_{+}^{2 *}$ foi estimado por meio de $S_{+}=\sqrt{1 / \sum_{i=1}^{k} \sum_{j=1}^{m_{i}}\left(1 / w_{i j}^{*}\right)}$ (Koots et al., 1994).

\section{Resultados e Discussão}

Na análise de agrupamento, usando raça, país, método de estimação, tamanho da amostra $(N)$ e $\hat{h}^{2}$, foram identificados, de acordo com o critério de pseudo F, quatro grupos para as características PN (pseudo F=201), P365 (pseudo F=213) e P550 (pseudo $\mathrm{F}=247$ ) e cinco grupos para a PD (pseudo $\mathrm{F}=405$ ) (Tabela 2).

Nos grupos formados, dentro das quatro características, verifica-se que, quando a porcentagem de animais da raça Nelore é alta, o país associado é o Brasil, sendo reflexo que o rebanho brasileiro analisado, de bovinos de corte zebuínos, é constituído, em
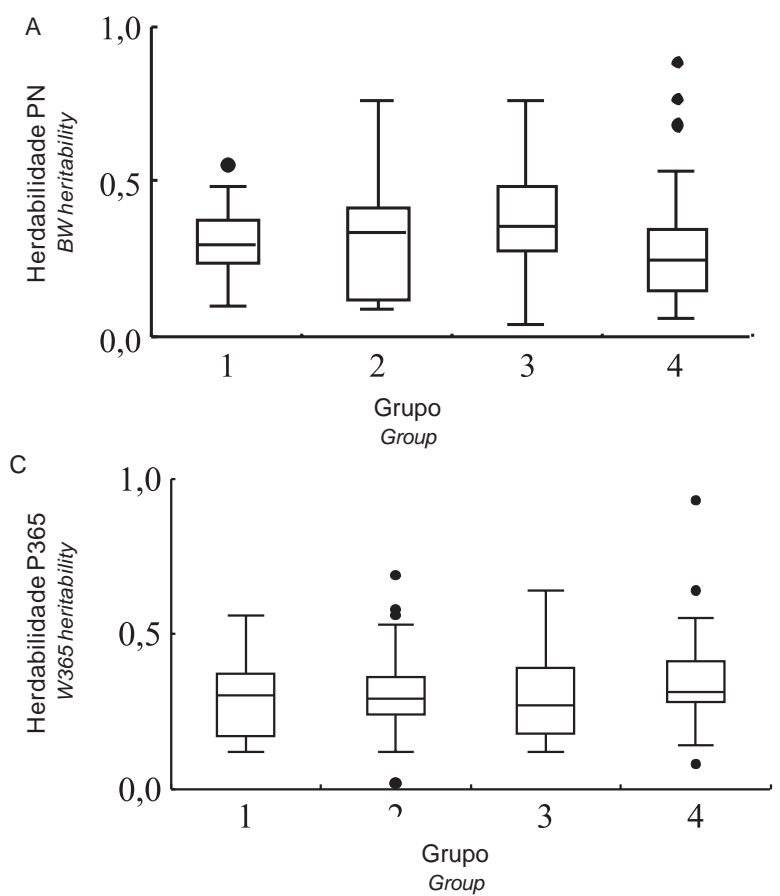

sua maioria, por animais da raça Nelore, corroborando as informações da Associação Brasileira de Criadores de Zebu (ABCZ, 2004) de que a raça Nelore representa cerca de $80 \%$ da força produtiva da indústria de carne no Brasil.

Ainda com relação aos grupos, verificou-se, em todas as características, que o modelo animal está sempre associado aos maiores valores de tamanhos amostrais médios ( $\bar{N}>4200$ ), enquanto as menores amostras estão associadas ao modelo touro ( $\bar{N}<3600$ ), o que pode ser explicado pelo aumento, no decorrer do tempo, das bases de dados de pesos dos animais e pela melhoria tecnológica ocorrida no final do século $\mathrm{XX}$, o que possibilitou maior capacidade de armazenagem e análise de grandes conjuntos de dados, com aplicação de modelos matemáticos mais sofisticados e biologicamente mais abrangentes.

Os box-plots (Figura 1) indicam a presença de discrepantes nos grupos: 1 e 4 da característica PN; 2, 4 e 5 da característica PD; 2 e 4 da característica P365; 1,2,e 3 da característica P550.

Os valores discrepantes podem influenciar a distribuição das $\hat{h}^{2}$, porém, apesar de terem sido detectados, foram mantidos na análise, posto que, nos trabalhos originais, os autores fazem considerações sobre as causas de terem obtidos valores elevados ou
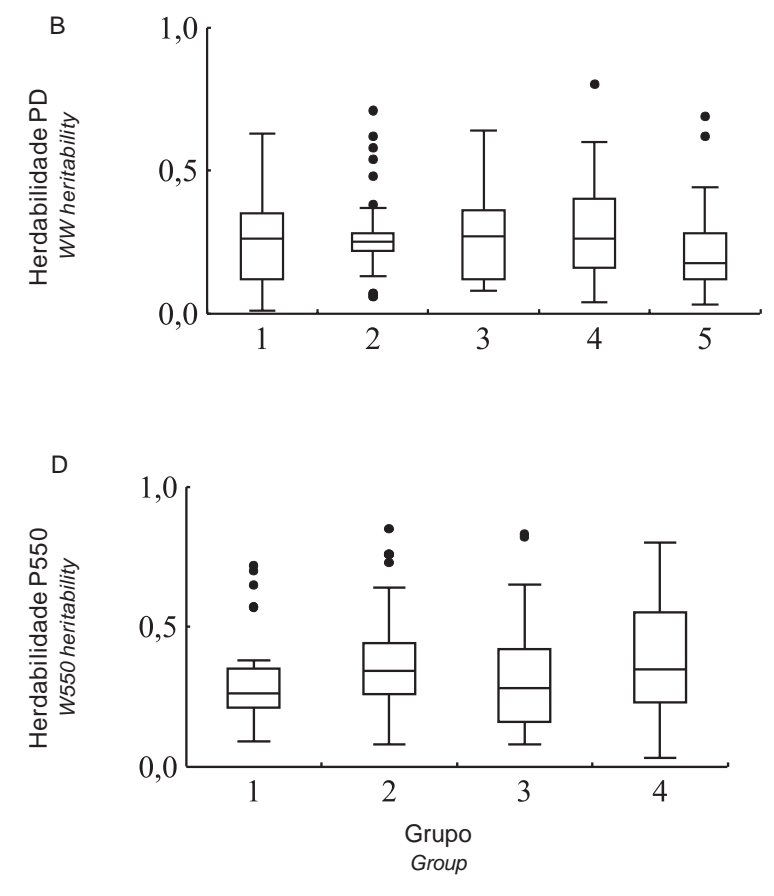

Figura 1 - Box-plots das estimativas de herdabilidade para os grupos dentro das características: (a) peso ao nascimento (PN); (b) peso à desmama (PD); (c) peso aos 365 dias (P365); e (d) peso aos 550 dias (P550).

Figure 1 - Box-plots of heritability estimates for groups within traits: (a) birth weight (BW), (b) weaning weight (WW), (c) weight at 365 days (W365); and (d) weight at 550 days (W550). 
Tabela 2 - Número de estimativas de herdabilidade $\left(\hat{h}^{2}\right)$, porcentagens das variáveis e tamanhos amostrais (mínimo, médio e máximo) presentes nos grupos dentro das características peso ao nascimento (PN), peso à desmama (PD), peso aos 365 dias (P365) e peso aos 550 dias (P550)

Table 2 - Number of heritability estimates $\left(\hat{h}^{2}\right)$, percentages of variables, and sample sizes (minimum, medium and maximum) in groups within the traits birth weight (BW), weaning weight (WW), weight at 365 days (W365) and weight at 550 days (W550)

\begin{tabular}{|c|c|c|c|c|c|c|c|c|c|}
\hline \multirow[b]{2}{*}{$\begin{array}{l}\text { Característica } \\
\text { Trait }\end{array}$} & \multirow[b]{2}{*}{$\begin{array}{l}\text { Grupo } \\
\text { Group }\end{array}$} & \multirow[b]{2}{*}{$\begin{array}{l}\text { № de } \hat{h}^{2} \\
N \text {. of } \hat{h}^{2}\end{array}$} & \multirow{2}{*}{$\begin{array}{c}\begin{array}{c}\text { Raça } \\
\text { Breed }\end{array} \\
\begin{array}{c}\text { Nelore }(\%) \\
\text { Nellore }\end{array} \\
\end{array}$} & \multirow{2}{*}{$\begin{array}{c}\text { País } \\
\text { Country } \\
\text { Brasil (\%) } \\
\text { Brazil }\end{array}$} & \multicolumn{2}{|c|}{$\begin{array}{l}\text { Modelo } \\
\text { Model }\end{array}$} & \multicolumn{3}{|c|}{$\begin{array}{l}\text { Tamanho amostral } \\
\text { Sample size }\end{array}$} \\
\hline & & & & & $\begin{array}{c}\text { MA (\%) } \\
A M\end{array}$ & $\begin{array}{l}\text { Touro (\%) } \\
\text { Sire }\end{array}$ & $\begin{array}{l}\text { Mín. } \\
\text { Min. }\end{array}$ & $\begin{array}{l}\text { Médio } \\
\text { Med. }\end{array}$ & $\begin{array}{l}\text { Máx. } \\
\text { Max. }\end{array}$ \\
\hline PN & 1 & 62 & 100 & 100 & 100 & 0 & 1019 & 20675 & 53429 \\
\hline \multirow[t]{3}{*}{$B W$} & 2 & 27 & 0 & 33 & 100 & 0 & 967 & 7473 & 41368 \\
\hline & 3 & 43 & 100 & 95 & 0 & 88 & 259 & 3212 & 28959 \\
\hline & 4 & 50 & 0 & 54 & 0 & 90 & 63 & 1572 & 31488 \\
\hline PD & 1 & 43 & 46 & 4 & 2 & 70 & 63 & 1956 & 31488 \\
\hline \multirow[t]{4}{*}{$W W$} & 2 & 434 & 100 & 100 & 100 & 0 & 737 & 21517 & 88412 \\
\hline & 3 & 61 & 0 & 41 & 100 & 0 & 712 & 7650 & 38845 \\
\hline & 4 & 47 & 100 & 100 & 0 & 100 & 171 & 2100 & 29032 \\
\hline & 5 & 46 & 0 & 100 & 0 & 100 & 222 & 2021 & 18751 \\
\hline P365 & 1 & 25 & 0 & 72 & 92 & 0 & 153 & 10294 & 28239 \\
\hline \multirow[t]{3}{*}{ W365 } & 2 & 78 & 100 & 100 & 100 & 0 & 848 & 13296 & 37004 \\
\hline & 3 & 29 & 100 & 100 & 0 & 100 & 87 & 3567 & 29032 \\
\hline & 4 & 21 & 0 & 62 & 0 & 100 & 63 & 2443 & 31488 \\
\hline P550 & 1 & 26 & 8 & 42 & 0 & 77 & 98 & 2141 & 31488 \\
\hline \multirow[t]{3}{*}{ W550 } & 2 & 85 & 100 & 100 & 100 & 0 & 871 & 14422 & 44075 \\
\hline & 3 & 50 & 0 & 36 & 100 & 0 & 264 & 4292 & 18493 \\
\hline & 4 & 42 & 100 & 100 & 0 & 100 & 87 & 1494 & 7102 \\
\hline
\end{tabular}

baixos para as $\hat{h}^{2}$, o que está de acordo com o procedimento adotado por Lôbo et al. (2000). Observa-se, graficamente, relativo alinhamento das caixas dos grupos, dentro das quatro características, o que possibilita a comparação entre eles. O teste de ShapiroWilk indicou que as $\hat{h}^{2}$ não seguem distribuição normal, havendo, pois, a necessidade de transformação pelo algoritmo de Box \& Cox (1964).

No teste de homogeneidade (Tabela 3), para as $\hat{h}^{2}$ transformadas entre e dentro dos grupos para as quatro características, os valores da estatística $Q$ sugerem a rejeição das hipóteses de nulidade em todos os níveis testados $(\mathrm{p} \leq 0,05)$, indicando a heterogeneidade tanto entre como dentro dos grupos.

A heterogeneidade entre os grupos era um resultado esperado, em virtude do método utilizado para o agrupamento; porém, almejava-se conseguir homogeneidade dentro de todos os grupos de estudo, pois uma das principais características do método de Ward é a composição de grupos bastante homogêneos, de acordo com os fatores utilizados para sua formação, mas a homogeneidade foi confirmada apenas para os grupos: 4 das características PN e P365; 5 da característica PD; e 1 da característica P550.
Sempre que a hipótese de homogeneidade foi satisfeita, adotou-se um modelo de efeito fixo para reunir as $\hat{h}^{2}$. Contudo, a solução proposta para reunir as $\hat{h}^{2}$, quando não satisfeita a hipótese de homogeneidade, foi modelar a variância existente entre as estimativas e incorporá-la ao modelo, adotando-se um modelo de efeito aleatório (Wang \& Bushman, 1999).

As variâncias entre os estudos, para as quatro características, estimadas pelos métodos $\operatorname{MVR}\left(\hat{\tau}_{M V R}^{2}\right)$ e $D L\left(\hat{\tau}_{D L}^{2}\right)$ resultaram em: $\hat{\tau}_{M V R}^{2}=0,0069$ e $\hat{\tau}_{D L}^{2}=$ 0,0057 para PN; $\hat{\tau}_{M V R}^{2}=0,0059$ e $\hat{\tau}_{D L}^{2}=0,0049$ para $\mathrm{PD} ; \hat{\tau}_{M V R}^{2}=0,0070$ e $\hat{\tau}_{D L}^{2}=0,0070$ para P365; e $\hat{\tau}_{M V R}^{2}=$ 0,0084 e $\hat{\tau}_{D L}^{2}=0,0077$ para P550. Verificou-se que as $\hat{\tau}^{2}$ apresentaram as menores estimativas quando calculadas pelo método $D L$.

DerSimonian \& Laird (1986), Normand (1999) e Brockwell \& Gordon (2001) realizaram, independentemente, meta-análises com artigos da área médica, utilizando diferentes estimadores da variância entre os estudos. Comparando os resultados alcançados nestes trabalhos, esses autores verificaram que os valores da variância entre os estudos obtidos por 
Tabela 3 - Estatística $Q$ para homogeneidade entre e dentro de grupos, com nível de significância e grau de liberdade (GL), para as estimativas de herdabilidade das características peso ao nascimento (PN), peso à desmama (PD), peso aos 365 dias (P365) e peso aos 550 dias (P550)

Table 3 - Q statistic for homogeneity between and within groups, with level of significance and degree of freedom (D.F.), for heritability estimates of traits birth weight (BW), weaning weight (WW), weight at 365 days (W365) and weight at 550 days (W550)

\begin{tabular}{|c|c|c|c|c|c|c|c|c|}
\hline \multirow{3}{*}{$\begin{array}{l}\text { Causas de } \\
\text { heterogeneidade } \\
\text { Sources of } \\
\text { heterogeneity }\end{array}$} & \multicolumn{6}{|c|}{$\begin{array}{c}\text { Características } \\
\text { Traits }\end{array}$} & & \\
\hline & \multicolumn{2}{|c|}{$\begin{array}{l}\text { PN } \\
B W\end{array}$} & \multicolumn{2}{|r|}{$\begin{array}{l}\text { PD } \\
W W\end{array}$} & \multicolumn{2}{|c|}{$\begin{array}{l}\text { P365 } \\
W 365\end{array}$} & \multicolumn{2}{|c|}{$\begin{array}{l}\text { P550 } \\
W 550\end{array}$} \\
\hline & $\mathrm{GL}(D F)$ & $Q$ & $\mathrm{GL}(D F)$ & $Q$ & $\mathrm{GL}(D F)$ & $Q$ & $\mathrm{GL}(D F)$ & $Q$ \\
\hline $\begin{array}{l}\text { Entre grupos } \\
\text { Between groups }\end{array}$ & 3 & $28,00 * *$ & 4 & $44,22^{* *}$ & 3 & $22,29 * *$ & 3 & $79,31^{* *}$ \\
\hline $\begin{array}{l}\text { Dentro de grupos } \\
\text { Within groups }\end{array}$ & 178 & $591,67 * *$ & 326 & $1160,96 * *$ & 149 & $646,83^{* *}$ & 199 & $664,71^{* *}$ \\
\hline $\begin{array}{l}\text { Dentro do grupo } 1 \\
\text { Within group } 1\end{array}$ & 61 & $276,88^{* *}$ & 42 & $73,12^{* *}$ & 24 & $138,15^{* *}$ & 25 & $22,97^{\mathrm{ns}}$ \\
\hline $\begin{array}{l}\text { Dentro do grupo } 2 \\
\text { Within group } 2\end{array}$ & 26 & $148,28 * *$ & 133 & $564,12 * *$ & 77 & $434,68 * *$ & 84 & $320,10 * *$ \\
\hline $\begin{array}{l}\text { Dentro do grupo } 3 \\
\text { Within group } 3\end{array}$ & 42 & $100,18^{* *}$ & 60 & $401,28 * *$ & 28 & $59,75^{* *}$ & 49 & $259,16 * *$ \\
\hline $\begin{array}{l}\text { Dentro do grupo } 4 \\
\text { Within group } 4\end{array}$ & 49 & $66,34^{\mathrm{ns}}$ & 46 & $73,46 * *$ & 20 & $14,26^{\mathrm{ns}}$ & 41 & $62,47 *$ \\
\hline $\begin{array}{l}\text { Dentro do grupo } 5 \\
\text { Within group } 5\end{array}$ & & & 45 & $48,98^{\text {ns }}$ & & & & \\
\hline Total & 181 & $619,65 * *$ & 330 & $1205,18^{* *}$ & 152 & $669,12 * *$ & 202 & $744,01 * *$ \\
\hline
\end{tabular}

ns Não-significativo pelo teste $Q(p>0,05)$ (Not significant by $Q$ test, $p>0.05)$.

* Significativo pelo teste $Q(p \leq 0,05)$ (Significant by $Q$ test, $p \leq 0.05)$.

** Significativo pelo teste $Q(p \leq 0,01)$ (Significant by $Q$ test, $p \leq 0.01$ ).

A

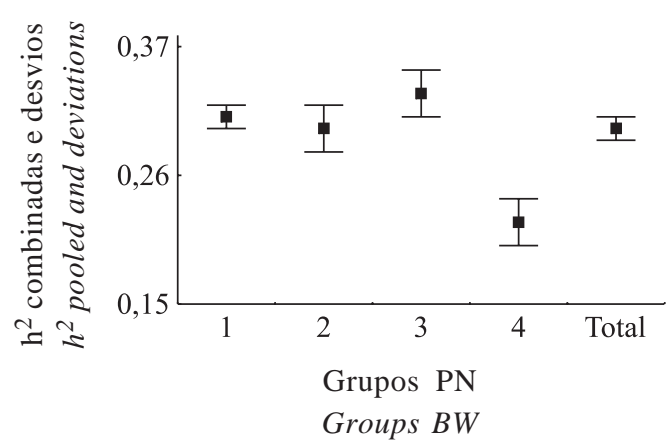

C

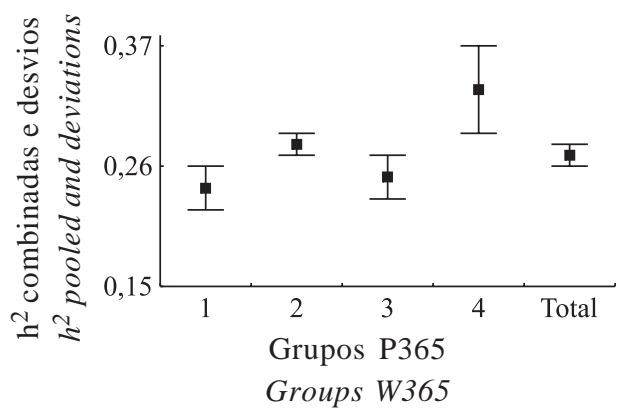

B

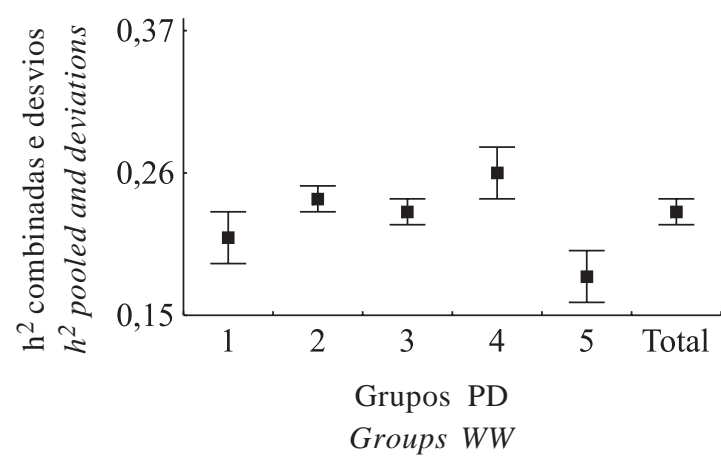

D

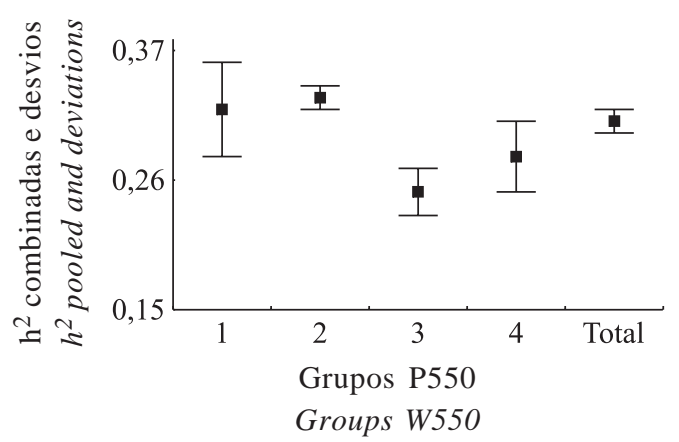

Figura 2 - Estimativas de herdabilidades combinadas por máxima verossimilhança restrita e desvio-padrão para as características: (a) peso ao nascimento (PN); (b) peso à desmama (PD); (c) peso aos 365 dias (P365); (d) e peso aos 550 dias (P550).

Figure 2 - Pooled heritability estimates by restricted maximum likelihood and standard deviation, for the traits: (a) birth weight (BW), (b) weaning weight (WW), (c) weight at 365 days (W365); and (d) weight at 550 days (W550). 
Tabela 4 - Estimativas de herdabilidades combinadas e desvios-padrão provenientes dos métodos da máxima verossimilhança restrita $\left(\hat{h}_{+M V R}^{2 *}\right)$ e o de DerSimonian e Laird $\left(\hat{h}_{+D L}^{2^{*}}\right)$

Table 4 - Pooled heritability estimates and standard deviations derived from methods restricted maximum likelihood and DerSimonian and Laird

\begin{tabular}{|c|c|c|c|c|c|c|c|c|}
\hline \multirow{3}{*}{$\begin{array}{l}\text { Grupo } \\
\text { Group }\end{array}$} & \multicolumn{8}{|c|}{$\begin{array}{c}\text { Característica } \\
\text { Trait } \\
\end{array}$} \\
\hline & \multicolumn{2}{|c|}{$\begin{array}{l}\text { Peso ao nascimento } \\
\text { Birth weight }\end{array}$} & \multicolumn{2}{|c|}{$\begin{array}{l}\text { Peso à desmama } \\
\text { Weaning weight }\end{array}$} & \multicolumn{2}{|c|}{$\begin{array}{l}\text { Peso aos } 365 \text { dias } \\
\text { Weight at } 365 \text { days }\end{array}$} & \multicolumn{2}{|c|}{$\begin{array}{l}\text { Peso aos } 550 \text { dias } \\
\text { Weight at } 550 \text { days }\end{array}$} \\
\hline & $\hat{h}_{+M V R}^{2^{*}}$ & $\hat{h}_{+D L}^{2 *}$ & $\hat{h}_{+M V R}^{2^{*}}$ & $\hat{h}_{+D L}^{2 *}$ & $\hat{h}_{+M V R}^{2^{*}}$ & $\hat{h}_{+D L}^{2 *}$ & $\hat{h}_{+M V R}^{2^{*}}$ & $\hat{h}_{+D L}^{2^{*}}$ \\
\hline 1 & $0,31 \pm 0,01$ & $0,31 \pm 0,01$ & $0,21 \pm 0,02$ & $0,20 \pm 0,02$ & $0,24 \pm 0,02$ & $0,24 \pm 0,02$ & $0,32 \pm 0,04$ & \\
\hline 2 & $0,30 \pm 0,02$ & $0,30 \pm 0,02$ & $0,24 \pm 0,01$ & $0,24 \pm 0,01$ & $0,28 \pm 0,01$ & $0,28 \pm 0,01$ & $0,33 \pm 0,01$ & $0,33 \pm 0,01$ \\
\hline 3 & $0,33 \pm 0,02$ & $0,33 \pm 0,02$ & $0,23 \pm 0,01$ & $0,23 \pm 0,01$ & $0,25 \pm 0,02$ & $0,25 \pm 0,02$ & $0,25 \pm 0,02$ & $0,25 \pm 0,02$ \\
\hline 4 & $0,22 \pm 0,02$ & $0,26 \pm 0,02$ & $0,26 \pm 0,02$ & $0,33 \pm 0,04$ & $0,28 \pm 0,03$ & $0,28 \pm 0,03$ & & \\
\hline 5 & & & $0,18 \pm 0,02$ & & & & & \\
\hline Total & $0,30 \pm 0,01$ & $0,30 \pm 0,01$ & $0,23 \pm 0,01$ & $0,23 \pm 0,01$ & $0,27 \pm 0,01$ & $0,27 \pm 0,01$ & $0,31 \pm 0,01$ & $0,31 \pm 0,01$ \\
\hline
\end{tabular}

$M V R$ foram sempre superiores aos obtidos por $D L$, corroborando os encontrados no presente trabalho.

Para obtenção das estimativas combinadas das herdabilidades $\left(\hat{h}_{+}^{2 *}\right)$, e seus respectivos desvios-padrão, utilizaram-se as $\hat{h}^{2}$ transformadas, mas os resultados estão apresentados na escala original. A incorporação das $\hat{\tau}^{2}$ obtidas anteriormente nas ponderações ocorreu apenas nos grupos em que a homogeneidade entre os estudos não foi confirmada. Entretanto, para os grupos: 4 das características PN e P365; 5 da característica PD; e 1 da característica P550, utilizou-se como ponderação o inverso da variância da própria $\hat{\tau}^{2}$ (Tabela 4; Figura 2).

Nos grupos em que foram incluídas as $\hat{\tau}^{2}$ na ponderação, constatou-se que os valores das $\hat{h}_{+}^{2^{*}}$, provenientes dos dois métodos de estimação, foram próximos, mesmo observando-se que, quando se consideram-se cada uma das quatro características em estudo, os valores das estimativas das $\hat{\tau}^{2}$ obtidas pelo método de $D L$ foram mais baixos dentro e entre os grupos.

Quanto à magnitude obtida para as $\hat{h}_{+}^{2 *}$, nos grupos formados nas quatro características, os valores foram mais baixos que os considerados referências por Bourdon (2000), de 0,40 para PN; 0,30 para PD; 0,40 para P365; e 0,65 para P550. São consideradas pouco herdáveis estimativas com valores abaixo de 0,20, moderadamente herdáveis estimativas com valores entre 0,20 e 0,40 e altamente herdáveis estimativas com valores superiores a 0,40 (Bourdon, 2000).
Assim, as estimativas combinadas obtidas neste trabalho podem ser consideradas moderadamente herdáveis para todos os grupos, exceto para o grupo 5 da característica PD, em que encontrou-se um valor mais baixo.

As maiores $\hat{h}_{+}^{2 *}$ foram encontradas em grupos compostos, na sua maioria, por animais da raça Nelore com dados originados no Brasil, refletindo a grande variabilidade genética de bovinos Nelore para as características de crescimento, o que permite que ganhos genéticos satisfatórios possam ser atingidos em programas de melhoramento que incluam estas características em seu critério de seleção.

A formação de grupos de resultados com mesmas características, como realizado neste trabalho, empregando-se a técnica de agrupamento, pode ser interessante para o usuário das estimativas de herdabilidade combinada, uma vez que esta técnica permite fornecer valores específicos, com características específicas e mais próximas da realidade, em vez de uma única estimativa combinada geral, como mostrado nas revisões de Mercadante et al. (1995), Koots et al. (1994) e Lôbo et al. (2000). Portanto, se é necessário ter, por exemplo, uma estimativa combinada de peso ao nascer proveniente de animais somente da raça Nelore, usando exclusivamente o modelo animal, a partir das informações contidas na Tabela 2 e na Figura 2, opta-se por usar o valor estimado no grupo 1. 


\section{Conclusões}

Apesar de os valores das estimativas da variância entre os estudos terem sido distintos nos dois métodos de estimação, os valores da estimativa combinada das herdabilidades, nas quatro características, foram próximos, independentemente do método utilizado.

A análise de agrupamento permitiu a formação de grupos de resultados de estudos semelhantes, fornecendo, assim, estimativas combinadas de herdabilidades para grupos específicos.

\section{Agradecimento}

Ao Conselho Nacional de Desenvolvimento Científico e Tecnológico (CNPq), pelo financiamento e apoio ao desenvolvimento deste estudo.

\section{Literatura Citada}

ASSOCIAÇÃO BRASILEIRA DE CRIADORES DE ZEBU. Raças zebuínas. http://www.braziliancattle.com.br/ racaszebuinas.php. (10 fev. 2004).

BOURDON, G.E.P. Understanding animal breeding. Upper Saddle River: Prendice-Hall, 2000. 538p.

BOX, G.E.P.; COX, D.R. An analysis of transformations. Journal of the Royal Statistical Society, v.26, p.211-252, 1964.

BROCKWELL, S.E.;GORDON, I.R. A comparation of statistical methods for meta-analysis. Statistics in Medicine, v.20, p.825-840, 2001.

BUSSAB, W.O.; MORETTIN, P.A. Estatística básica. 5.ed. São Paulo: Saraiva, 2003. 526p.

DerSIMONIAN, R.; LAIRD, N. Meta-analysis in clinical trials. Controlled Clinical Trials, v.7, p.177-188, 1986.

FAGARD, R.H.; STAESSEN, J.A.; THIJS, L. Advantages and disadvantages of the meta-analysis approach. Journal of Hypertension, v.14, suppl.2, p.9-13, 1996.

FALCONER, D.S.; MACKAY, T.F.C. Introduction to quantitative genetics. Edinburgh: Addison Wesley Longman, 1996. 464p.

GLASS, G.V. Primary, secundary, and meta-analysis of research. Educational Researcher, v.6, p.3-8, 1976.

HEDGES, L.V.; OLKIN, I. Statistical methods for metaanalysis. London: Academic Press, 1985. 369p.

JOHNSON, R.A.; WICHERN, D.W. Applied multivariate statistical analysis. 4.ed. Englewood Cliffs: Prentice Hall, 1998. 816p.
KOOTS, K.R.; GIBSON, J.P.; SMITH, C. et al. Analyses of publised genetic parameter estimates for beef production traits. 1. Heritability. Animal Breeding Abstracts, v.62, n.5, p.309-338, 1994.

LÔBO, R.N.B; MADALENA, F.E.; VIEIRA, A.R. Average estimates of genetic parameters for beef and dairy cattle in Tropical regions. Animal Breeding Abstracts, v.68, p.433-462, 2000.

MERCADANTE, M.E.Z.; LÔBO, R.B.; REYES, A. Parámetros genéticos para características de crecimiento em cebuínos de carne: una revisión. Archivo Latinoamericano Producción Animal, v.3, n.1, p.45-89, 1995.

NORMAND, S.T. Tutorial in biostatistics meta-analysis: formulating, evaluating, combining, and reporting. Statistics in Medicine, v.18, p.321-359, 1999.

STATISTICAL ANALYSES SYSTEM - SAS. SAS/IML software usage and reference, version 6. Cary: 1990. 510p.

STATISTICAL ANALYSES SYSTEM - SAS. SAS/STAT software: changes and enhancements through release 6.07. 6.12. Cary: 1997. 1162p.

STATISTICAL ANALYSES SYSTEM - SAS. SAS macro programs: boxcox - power transformations by Box-Cox method. 2002. http://www.math.yorku.ca/SCS/sasmac/ boxglm.html. (04 ago. 2003)

STATISTICAL ANALYSES SYSTEM - SAS. SAS OnlineDoc ${ }^{\circledR}$ : version 8. Cary. 1999a. http:// www.id.unizh.ch/software/unix/statmath/sas/sasdoc/stat/ chap8/sect10.htm. (21 nov. 2003).

STATISTICAL ANALYSES SYSTEM - SAS. SAS OnlineDoc ${ }^{\circledR}$ : version 8. Cary: 1999b. http:// www.id.unizh.ch/software/unix/statmath/sas/sasdoc/stat/ chap23/sect25.htm. (21 nov. 2003).

STATISTICAL ANALYSES SYSTEM - SAS. SAS OnlineDoc ${ }^{\circledR}$ : version 8. Cary: 1999c. http:// www.id.unizh.ch/software/unix/statmath/sas/sasdoc/stat/ chap23/sect2.htm. (21 nov. 2003).

SHAPIRO, S.S.; WILK, M.B. An analysis of variance test for normality (complete samples). Biometrika, v.52, p.591611, 1965.

SHARMA, S. Applied multivariate techniquies. NewYork: John Wiley, 1996. 493p.

WANG, M.C.; BUSHMAN, B.J. Integration results: through meta-analytic review using SAS software. Cary: SAS Institute, 1999. 400p. 